\title{
Measurement of the Top Quark Mass at CDF
}

\author{
CDF Collaboration
}

Presented by Steve Vejcik

University of Michigan

Ann Arbor, MI 48109

\begin{abstract}
We report the measurement of the Top Quark using complete kinematic reconstruction of events with a $W$ and 4 jets. Included in this talk are a brief description of the technique, the observed results when applied to events before and after requiring a b-tagged jet, and discussion of systematic aspects of the measurement. We additionally present results which indicate the presence of a second $W$ in the events decaying to two jets.
\end{abstract}

\section{INTRODUCTION AND TECHNIQUE}

The general technique used to reconstruct Top events revolves around the hypothesis that the observed events stem from the decay sequence:

$$
\begin{aligned}
& p \bar{p} \rightarrow t \bar{t} X \\
& \rightarrow W^{+} b W^{-\bar{b}} \\
& \rightarrow 1^{+} \nu b j j \bar{b}
\end{aligned}
$$

There are 13 four-momenta involved and therefore 52 variables. Using the known masses and energy-momentum conservation at each vertex results in an overconstrained system: there are two more known quantities than there are variables and we therefor have what is commonly referred to as a $2 \mathrm{C}$-Fit.

Since it is not known a priori what the flavor of the observed jets are, all possible jet-flavor assignments are independently fit to the above hypothesis. There are twelve possible configurations and often 24 since there is a possible quadratic ambiguity in the neutrino $z$-momentum, where $z$ is the direction of the incoming proton beam. Of the different combinations, the one with the best fit (lowest $\chi^{2}$ ) is taken to be the solution and an accepted solution is required to have a $\chi^{2}<10.0$.

\section{IET RECONSTRUCTION AND CORRECTIONS}

In the event topology studied, most of the final state particles result in an observed jet and the accurate measurement of the jet momenta is therefore 
important for obtaining a precise mass determination. With the fixed cone size clustering employed at $\mathrm{CDF}$, four components in a jet system can be identified: the original parton, the underlying event, the portion of a jet which lies within the clustering cone ${ }^{1}$, and the portion of the jet which lies outside of the cone. The measurement of the jet $\mathbf{E}_{\mathrm{T}}$ inside the jet cone is based on extensive studies at CDF based primarily on in situ calibration using charged particle tracks. For values of jet $E_{T}$ typical of Top decays, the uncertainty in the inner cone $\mathrm{E}_{\mathrm{T}}$ is $\approx 2-4 \%$. The goal of reconstructing Top decays is to obtain an estimate of the four momentum of the original partons. Beginning with the Jet $\mathrm{E}_{\mathrm{T}}$ inside the clustering cone, two additional corrections are used to estimate the parton momenum. The first correction employs Monte Carlo studies to estimate the portion of the Jet $\mathbf{E}_{\mathrm{T}}$ which lies outside the cone. Tests of these corrections are afforded by studies of the $\mathrm{E}_{\mathrm{T}}$ balance between events with either a $Z$ or $\gamma$ and a single recoiling jet.

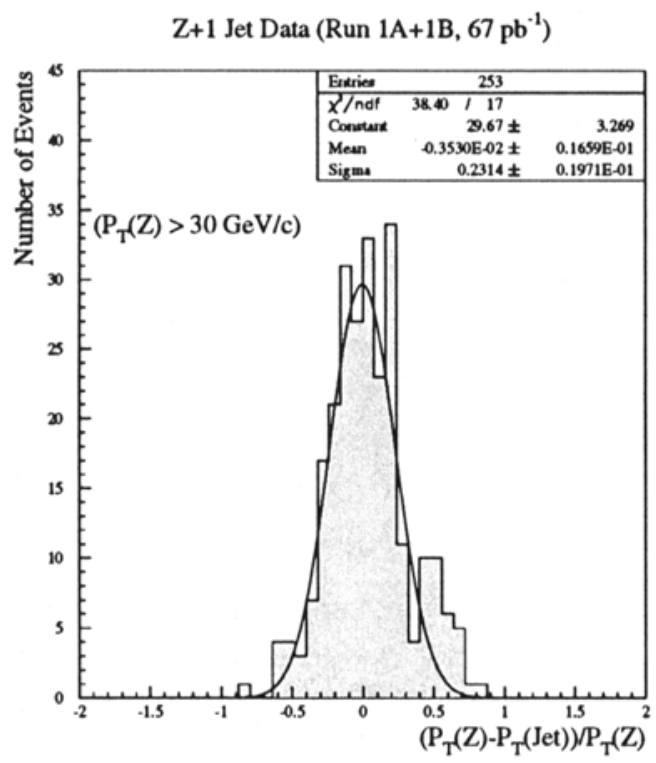

FIG. 1. $\mathrm{E}_{\mathrm{T}}$ balance between $Z$ and single jet events. The curve is a gaussian fit to the data.

Figure 1 shows the the results of the study for $Z+1$ Jet events indicating that the corrections achieve the expected balance to $\approx 3 \%$. Additional understanding of the jet $E_{T}$ outside the clustering cone comes from examing the $E_{T}$ in an annular region around a jet in both $Z$ and $W+1$ jet events. The annular

\footnotetext{
${ }^{1}$ In this analysis CDF has employed a cone size of $\Delta R=0.4$ where $\mathrm{R}$ is define as $\sqrt{\left((\Delta \eta)^{2}+(\Delta \phi)^{2}\right)}$
} 
region is chosen so that both the Monte Carlo derived outer cone corrections and the better understood inner cone corrections can be applied. Subsequent corrections estimate the parton momentum based on the fully corrected jets and therefore the test provided by the annulus study is whether the difference in the two types of corrections (the Monte Carlo derived corrections and the inner cone corrections) behave the same for Monte Carlo samples and for data. Figure 2 shows the comparison for $\mathrm{W}+1$ jet events and indicates a descrep-

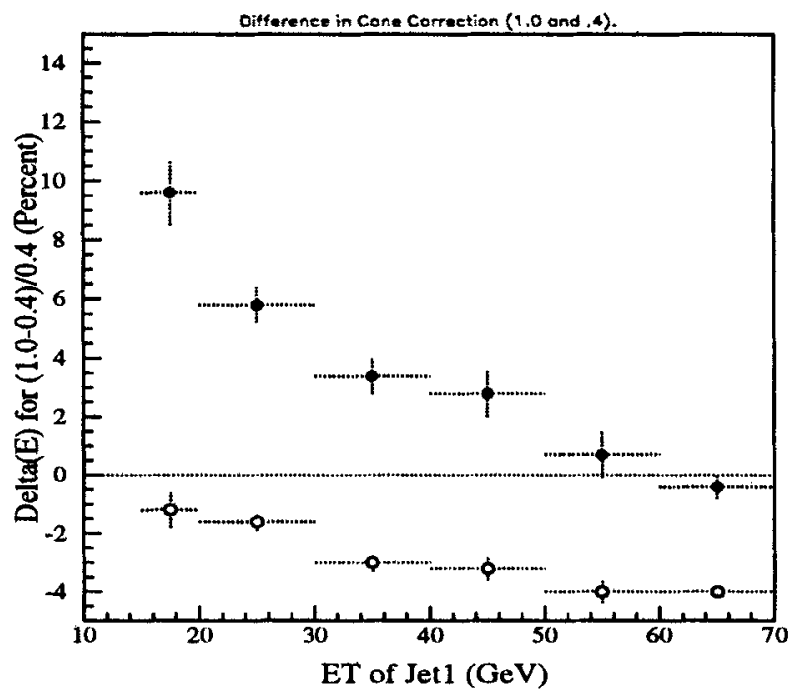

FIG. 2. Fractional difference in the $\mathrm{E}_{\mathrm{T}}$ in an annulus between $R=0.4$ and $R=1.0$ for $W+1$ jet events.

ancy at the $10 \%$ level at low $\mathrm{E}_{\mathrm{T}}$ decreasing to a few percent in the regime more typical for jets from Top decays. Based on these studies, it is therefore probable that the $4.4 \%$ uncertainty in the top mass presently cited (2), (1) ,due to the effects of soft radiation on the Jet $\mathrm{E}_{\mathrm{T}}$ scale, is an overestimate. As stated above, CDF also employs an additional set of jet corrections which account for features specific to Top decays. These correct for the presence of neutrinos and muons which occur in a large fraction of the decays of the associated $b$ hadrons produced in the Top decays and for effects of the Jet momentum distribution. Figure 3 shows the size of these corrections for different types of identified Jets. Finally Figure 4 shows the results of applying the algorithm and all corrections described to Monte Carlo generated samples of Top and the dominant background (QCD production of W+multijets). It can be seen in this figure that given the presence of a heavy Top quark, we expect to reconstruct its decay and observe a mass distribution which peaks near its true mass value. 


\section{RESULTS}

The data sample from which the Top quark mass is estimated is substantially the same as that used

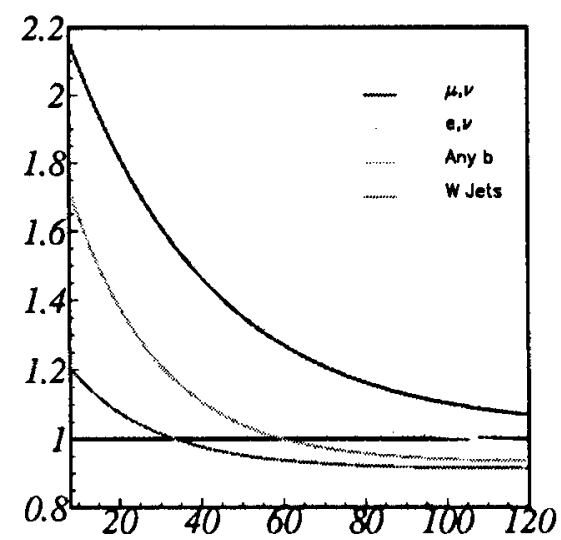

FIG. 3. Final corrections applied to jets from Top decays used to estimate the original parton momentum.

in the counting experiment (3). Specifically we require events with a cleanly identified $W$ decaying to either $\mu \bar{\nu}$ or $e \bar{\nu}$ and at least three jets with uncorrected $\mathrm{E}_{\mathrm{T}}>15 \mathrm{GeV}$. Since the event reconstruction algorithm requires at least four jets we further require a fourth jet with a looser $(>8 \mathrm{GeV}) \mathrm{E}_{\mathrm{T}}$ cut to retain a reasonable acceptance. This event selection results in a sample of 99 events. Applying the reconstruction algorithm to these events and requiring the resultant $\chi^{2}<10.0$ leaves 88 events whose mass distribution is shown in It can be seen that this distribution is not well modeled by the distribution expected from only background events, the disagreement being greatest in the vicinity of $160-190 \mathrm{GeV}$. To further substantiate the hypothesis that the disagreement is due to Top events, the subsample of events with at least one b-tagged (using either the SVX or SLT algorithms (3)) jet is examined. Figure 5. Given an event with a b-tagged jet, the mass reconstruction 
Reconstructed mass for HERWIG MonteC arlo, $\mathrm{M}_{\iota p}=170 \mathrm{GeV}$

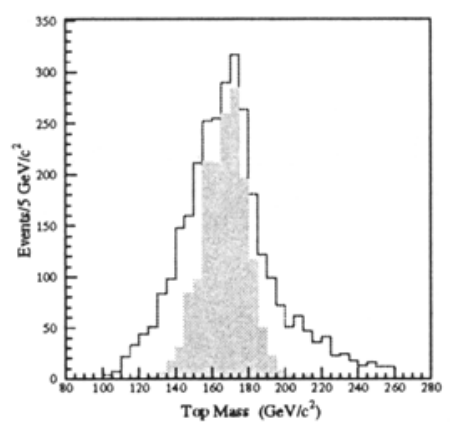

Reconstructed mass for the VECBOS+HERPRT $W+3$ jets events.

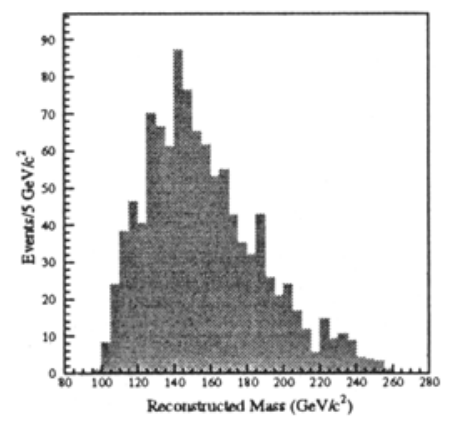

FIG. 4. Results of applying mass reconstruction algorithm to Monte Carlo samples of Top and the dominant background, QCD production of $W+$ multijets. 


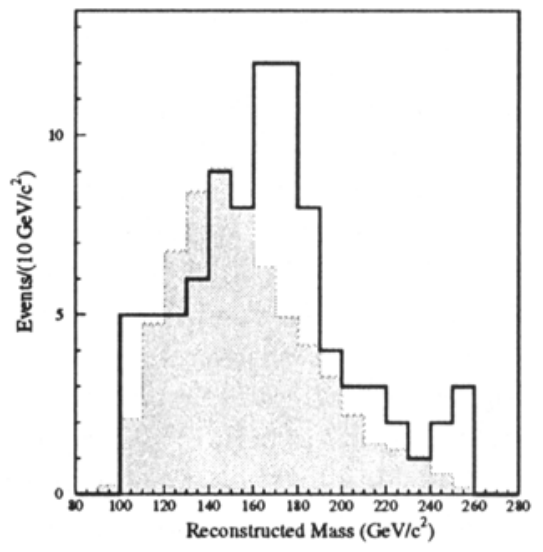

FIG. 5. Reconstructed mass distribution for 88 event sample of $W+4$ jet events. The shaded histogram is that expected for background with the normalization taken from a fit made with a background constraint.

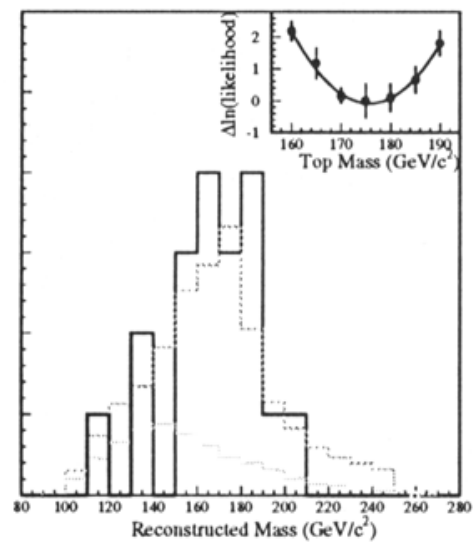

FIG. 6. Reconstructed mass distribution for events where at least one b-tag was observed. The dashed histograms represent the fitted distributions from background and the sum of background and signal. The inset plot shows the likelihood for similar fits to different Top mass hypotheses.

algorithm is modified so that only solutions which are consistent with the btagged jet corresponding to a b-parton are used. The resulting distribution of reconstructed mass for these events is shown in Figure 6. It can be seen that 
the events which have the additional characteristics expected for top decaysthe presence of $b$-quarks- have a much more localized distribution than that expected from the dominant background. The observed mass distribution can be used to measure $M_{T O P}$ by comparing it to different hypothesized top mass distributions. For each hypothesis, the observed distribution is fit to a combination of background and signal events including the estimated size of the background. The inset plot of Figure 6 shows the obtained likelihood for different mass hypotheses with a fit used to obtain the measured Top mass of $176 \pm 8 \mathrm{GeV} / \mathrm{c}^{2}$ where the uncertainty is statistical.

As a further check that the events originate from the decay chain cited earlier, we have looked for evidence of a second $\mathrm{W}$ decaying to two jets using two techniques. In the first technique, the invariant mass distribution is formed for all six dijet combinations resulting from the four highest $E_{T}$ jets. To reduce the combinatorics, a cut is made on $\mathrm{H}$, the total visible energy for an event. Studies (4) have indicated that events with large values of $H$ improve the fraction of Top events in the event sample. Figure 7 shows the

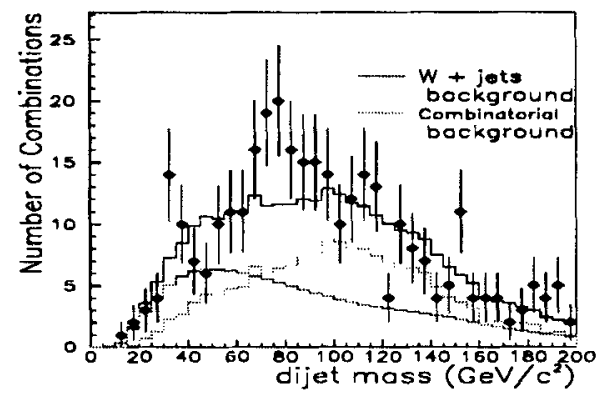

FIG. 7. Dijet mass distribution for $W+4$ jet events. All dijet combinations using the four highest $E_{T}$ are included and events were required to have total visible energy (H) $>300 \mathrm{GeV}$.

dijet mass distribution with the additional requirement that $H>300 \mathrm{GeV}$ together with the dijet distribution expected from the dominant backgroud process and the distribution expected from the combinatoric background of Top events. A clear excess above these two components is observed in the range of $M_{J J} \approx 80 \mathrm{GeV} / \mathrm{c}^{2}$ as expected for Standard Model Top decays. An second way to look for the hadronically decaying $W$ is to modify the event reconstruction by removing the constraint that two jets originate from a $\mathrm{W}$ and refit the event. The invariant mass of the two jets associated by this procedure with the decaying $W$ is plotted in Figure 8. Again, a clear excess above the distribution expected from the background is observed in the region of $M_{J J} \approx 80 \mathrm{GeV} / \mathrm{c}^{2}$. We conclude that the observed events from which we measure the Top mass show kinematic evidence of a second $\mathrm{W}$ as expected 
from our model of the Top decays.

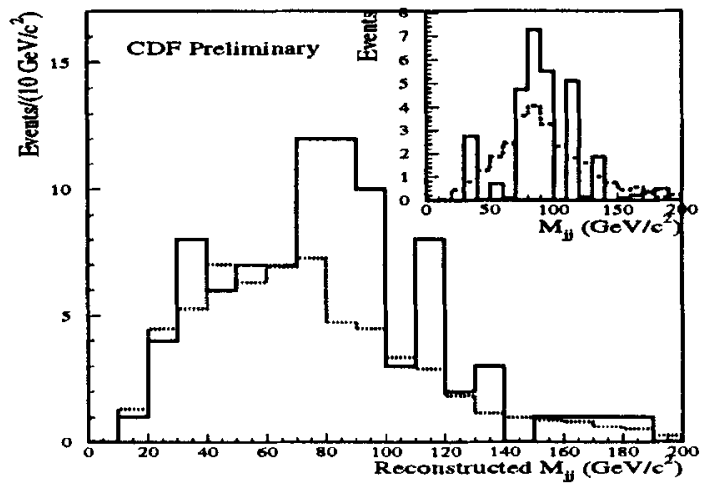

FIG. 8. Observed distribution of dijet invariant mass using the two jets identified by the Top mass reconstruction algorithm as being associated with the decay $W \rightarrow j j$. The algorithm did not use the constraint that the dijet invariant mass equal that of the $W$ boson.

\section{SYSTEMATICS}

The uncertainty in the measured Top quark mass is currently dominated by systematic effects. Understanding each aspect is the current focus of efforts within the CDF collaboration and the the current estimates are substantially unchanged from those reported with CDF's first observation of evidence for Top production (1). Two systematics, however, bear some comment. First, the dominant uncertainty is currently our understanding of the effects of gluon radiation on our Jet $\mathrm{E}_{\mathrm{T}}$ scale. As discussed above, this uncertainty is now believed to be an overestimate and will be reduced in the near future. It can also be noted that the systematic quoted in (1) which reflected our lack of knowledge of the mass distribution for non- $W+$ jet background events has been removed. The event reconstruction algorithm has been applied to both Monte Carlo and data samples of $Z+4$ Jets, Non-isolated Lepton +4 Jets, and non-central Lepton +4 Jets. In each case, the resulting mass distribution is observed to be consistent with the mass distribution obtained from the expected dominant background of QCD produced W+multijets. As an example, 
Figure 10 shows the distribution obtained from reconstructing $Z+4$ Jet events compared with Monte Carlo distributions of $Z+4$ Jet and $W+4$ Jet events. Retaining the remaining systematic uncertainties from the analysis presented in (1), we estimate a total systematic uncertainty in the measurement of $M_{\text {TOP }}$ of $9.8 \mathrm{GeV} / \mathrm{c}^{2}$ or $5.6 \%$.

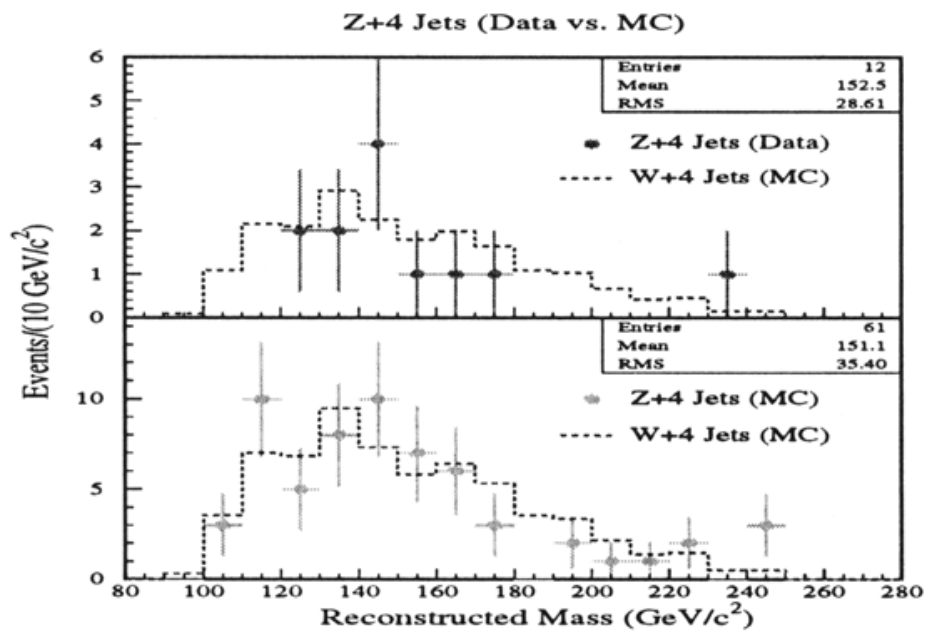

FIG. 9. Mass distribution for $Z+4$ jet events. The upper plot includes the distributions for events from Monte Carlo as well as data. The lower plot compares the Monte Carlo distribution for $Z+4$ jet events with that from $W+4$ jet Monte Carlo.

\section{CONCLUSION}

Using the same data sample for which we have demonstrated the existence of Top decays, we observed a mass distribution consistent with that expected for a Top quark with a mass in the $160-190 \mathrm{GeV}$ range. The same events have the additional feature of having evidence for a second hadronically decaying $W$ as expected for Standard Model Top decays. From the reconstructed Top mass distribution for events with at least one b-tag, we have estimated the Top quark mass to be $176 \pm 8 \pm 9.8 \mathrm{GeV} / \mathrm{c}^{2}$. Ongoing studies indicate that the quoted systematic uncertainty is likely to be an overestimate and is expected to be reduced in the near future. 


\section{ACKNOWLEDGMENTS}

We wish to thank the organizers of the conference for both the hospitality of the conference and the scientific environment provided.

\section{REFERENCES}

1. F. Abe et al., Phys. Rev. D 45, 1776 (1994)

2. F. Abe et al. Phys. Rev. Letters bf 73, 4330 (1995)

3. J. Incandela, These proceedings

4. M. Binkley, These proceedings 